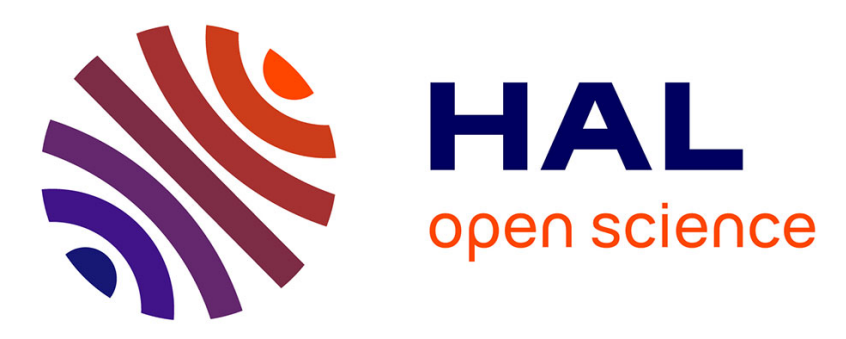

\title{
2D Sequence-Coded Oligourethane Barcodes for Plastic Materials Labeling
}

Denise Karamessini, Salomé Poyer, Laurence Charles, Jean-François Lutz

\section{To cite this version:}

Denise Karamessini, Salomé Poyer, Laurence Charles, Jean-François Lutz. 2D Sequence-Coded Oligourethane Barcodes for Plastic Materials Labeling. Macromolecular Rapid Communications, 2017, 38 (24), pp.1700426. 10.1002/marc.201700426 . hal-01959034

\section{HAL Id: hal-01959034 https://hal.science/hal-01959034}

Submitted on 18 Dec 2018

HAL is a multi-disciplinary open access archive for the deposit and dissemination of scientific research documents, whether they are published or not. The documents may come from teaching and research institutions in France or abroad, or from public or private research centers.
L'archive ouverte pluridisciplinaire HAL, est destinée au dépôt et à la diffusion de documents scientifiques de niveau recherche, publiés ou non, émanant des établissements d'enseignement et de recherche français ou étrangers, des laboratoires publics ou privés. 
This is the peer reviewed version of the following article:

Denise Karamessini, Salomé Poyer, Laurence Charles, * Jean-François Lutz*

Macromol Rapid Commun. 2017; 38, 1700426

which has been published in final form at

https://onlinelibrary-wiley-com.scd-rproxy.u-strasbg.fr/doi/epdf/10.1002/marc.201700426

This article may be used for non-commercial purposes in accordance with Wiley Terms and Conditions for Self-Archiving

\title{
2D sequence-coded oligourethane barcodes for plastic materials labeling
}

\author{
Denise Karamessini ${ }^{1}$, Salomé Poyer ${ }^{2}$, Laurence Charles, $^{2} *$ Jean-François Lutz ${ }^{1}$. \\ ${ }^{1}$ Université de Strasbourg, CNRS, Institut Charles Sadron UPR22, 23 rue du Loess, 67034 \\ Strasbourg Cedex 2, France._E-mail: jflutz@unistra.fr ${ }^{2}$ Aix-Marseille Université, CNRS, \\ UMR 7273, Institute of Radical Chemistry, 13397, Marseille Cedex 20, France._Email: \\ laurence.charles@univ-amu.fr
}

\begin{abstract}
Mixtures of uniform sequence-defined oligourethanes were evaluated as 2D molecular barcodes for labeling three different commodity polymers, namely polystyrene, polyvinylchloride and polyethylene terephthalate. Six different oligourethanes were synthesized by solid-phase iterative synthesis and were coded using a binary monomer alphabet.High-resolution mass spectrometrystudies indicated that all oligomers are uniform and sequence-defined. However, instead of using them as individual codedchains, oligomers with different chain-length mass and sequence were mixed into intentionally polydisperse libraries. In particular, a three-component and a four-component library were created to encode a 2-bytes model binary sequence. These 2D-coded libraries were incorporated in all commodity plastics via a simple solvent casting procedure. Furthermore, in all cases, the oligomer mixtures could be extracted from the host polymer films and deciphered by mass spectrometry, thus opening interesting avenues for anti-counterfeiting and traceability applications.
\end{abstract}




\section{Introduction}

Among the potential applications of sequence-controlled polymers, ${ }^{[1]}$ it has been suggested that anti-counterfeiting technologies could be an interesting area of use. ${ }^{[2]}$ Indeed, a macromolecule containing a perfectly defined sequence of monomers can potentially be used as a label or "barcode", ${ }^{[3]}$ in which molecular information certifying authenticity can be included. For instance, DNA, which contains a defined sequence of nucleotides, is sold by several companies as an anti-counterfeiting solution for the labeling of high-value materials. Since DNA strands can be selectively hybridized and amplified by polymerase chain reaction, DNA tags can be used in trace amounts for materials labeling. However, DNA is not stable in all conditions and may degrade during materials processing, e.g. in hot-melt extrusion, thermoset curing, photo-curing, 3D printing or sol-gel process. Thus, for many applications, DNA barcodes have to be encapsulated, for example in processable nanoparticles as developped by Grass and coworkers. ${ }^{[4-5]}$ Alternatively, non-natural sequence-defined polymers may also be used as anti-counterfeiting barcodes. ${ }^{[2]}$ Over the last years, we ${ }^{[6-11]}$ and others $^{[12-15]}$ have demonstrated that a wide variety of uniform sequence-defined synthetic macromolecules can be synthesized using multi-step growth approaches. ${ }^{[16]}$ In particular, our group has evidenced that such polymers can be used to store information. For instance, binary information can be easily written in synthetic sequence-defined macromolecules using two monomers that are defined as 0 and 1-bits. ${ }^{[17]}$ In addition, the digital sequences can be easily read using a sequencing methodology ${ }^{[18]}$ such as tandem mass spectrometry (MS/MS). ${ }^{[19-21]}$

Among all the different types of digital polymers that have been reported by our group, sequence-coded oligourethanes (or oligocarbamates) appear as a very interesting class of polymers for anti-counterfeiting technologies. Uniform sequence-defined oligocarbamates can be synthesized using a variety of solid-phase iterative approaches. ${ }^{[14,22-24]}$ In a publication reported last year, ${ }^{[25]}$ our group has shown that digital oligourethanes can be synthesized using a simple protecting-group-free iterative approach. ${ }^{[26]}$ Furthermore, it was found in this study 
that the $\mathrm{COOH}$-terminated oligomers prepared via this orthogonal approach are particularly easy to decode by negative-mode MS/MS sequencing. ${ }^{[25]}$ In other words, sequence-defined oligourethanes can easily be both coded and decoded. Furthermore, classical polyurethanes constitute a well-known class of plastics with widely-described physico-chemical properties, including for example chemical resistance and thermal stability. Therefore, sequence-coded oligourethanes appear as ideal candidates for materials labeling. For instance, we have already demonstrated that oligourethane barcodes can be included in small amounts (typically less than a weight percent) in casted polystyrene films, ${ }^{[25]} 3 \mathrm{D}$-printed photo-cured methacrylate resins $^{[25]}$ and acrylate-based intraocular implants. ${ }^{[27]}$ In all cases, it was also demonstrated that the barcodes can be selectively extracted from the host polymer materials and sequenced by MS/MS. Yet, in all these examples, the information sequence was included in a single oligomer chain (i.e. 1D-coding). In another recent study, our group has shown that information can also be written in a set of oligomers of different mass (i.e. 2 D-coding) ${ }^{[28]}$ In such a strategy, digital information is stored in a library of chains. The electrospray mass spectrometry (ESI-MS) analysis of these mixtures leads to an intentionally-polydisperse spectrum (i.e. first dimension fingerprint) and the MS/MS sequencing of eachindividual peakof this mass distributiongives access to the sequence-information included in each oligomer (i.e. second dimension fingerprint). This concept is powerful because $(i)$ it does not require the synthesis of long coded oligomers and (ii) it gives access to an increased security level for anti-counterfeiting applications, as compared to standard 1D barcodes. However, this interesting 2D approach was not tested so far for materials labeling, mostly because the reported proof-of-concept was obtained with sequence-coded oligo(alkoxyamine amide)s, ${ }^{[28]}$ which are thermolabile and thus very fragile oligomers. ${ }^{[10]}$ Hence, in the present work, the 2Dcoding strategy was applied to sequence-defined oligourethanes and tested for the labelling of different commodity plastics such as polystyrene (PS), polyvinylchloride (PVC) and polyethylene terephthalate (PET). 


\section{Results and Discussion}

Two libraries of sequence-coded oligourethanes were used as 2D barcodes for the labelling of thin plastics films (Figure 1). Each oligomer was prepared via a solid-phase orthogonal iterative process, as described in an earlier publication. ${ }^{[25]}$ In this approach, two monomers are used as a binary alphabet. In the formed oligourethanes, a 1-bit corresponds to a methylated synthon, whereas a 0-bit corresponds to its non-methylated analogue (Figure 1).Combining this $14 \mathrm{Da}$ mass difference with the specific dissociation pattern of sequencedefined oligourethanes in the negative ion mode, the nature of these synthons as well as their respective locations along these oligomeric chains can readily be distinguished in MS/MS sequencing, as reported earlier. ${ }^{[25]}$

\section{Coded library 1:}

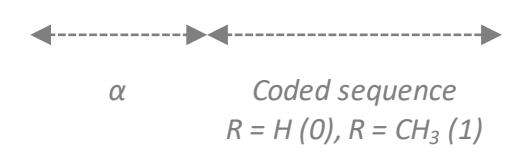

Bit $0=$

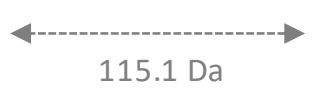

Bit $1=$
(P1) $\alpha-0-1$

(P2) $\alpha-$

(P3) $\alpha$ - (0)-0-1) (0)

(P4) $\alpha$-1)-(0)-(0)-(0)-1)-(0)

\section{Coded library 2:}

(P1) $\alpha-0$-1)

(P5) $\alpha-0-0-0$-0)-0) 1

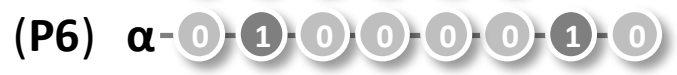

Figure 1. Sequence-coded oligourethane libraries used in this work for the labelling of plastic films. The general molecular structure of the oligourethanes appears on top left. Each oligomer is coded using a binary molecular alphabet. The structure and mass of the coded synthons are shown on bottom left. Two different libraries were used in this work: a fourcomponent library (top right) and a three-component library (bottom right). 
In the present work, the model digital sequence 0100000101000010 was encoded in oligurethanes. In order to show the versatility of the 2D barcoding concept, this sequence was written in two different libraries, containing either three of four components, as shown in Figure 1.The four components library contained the oligomers P1-P4 (Figure 1 and Table 1), with sequence $\alpha-01, \alpha-000, \alpha-0010$ and $\alpha-1000010$, to be combined from the smallest to the largest one in order to compose the model digital sequence. ${ }^{[28]}$ Alternatively, but using the same writing rules, ${ }^{[28]}$ this sequence was generated with oligomers from the three components library, namely P1, P5 and P6 (Figure 1 and Table 1), with sequence $\alpha-01, \alpha-000001$ and $\alpha-$ 01000010, respectively. In all oligomers, the prefix $\alpha$ in these sequences denotes a short spacer obtained after resin cleavage, as defined in previous works. ${ }^{[25,27]}$

Table 1.ESI-HRMS characterization of the sequence-coded oligourethanes. ${ }^{\text {a }}$

\begin{tabular}{llccc}
\hline & Sequence & Theoretical mass & Experimental Mass & Yields \\
& & $\boldsymbol{m} / \boldsymbol{z}_{\text {th }}$ & $\boldsymbol{m} / \boldsymbol{z}_{\text {exp }}$ & $(\%)$ \\
\hline P1 & $\alpha-0-1$ & 377.2282 & $377.2287^{\mathrm{b}}$ & 89 \\
$\mathbf{P 2}$ & $\alpha-0-0-0$ & 478.2759 & $478.2768^{\mathrm{b}}$ & 96 \\
$\mathbf{P 3}$ & $\alpha-0-0-1-0$ & 607.3549 & $607.3563^{\mathrm{b}}$ & 66 \\
$\mathbf{P 4}$ & $\alpha-1-0-0-0-0-1-0$ & 966.5605 & $966.5612^{\mathrm{b}}$ & 53 \\
$\mathbf{P 5}$ & $\alpha-0-0-0-0-0-1$ & 854.5081 & $854.5091^{\mathrm{c}}$ & 96 \\
$\mathbf{P 6}$ & $\alpha-0-1-0-0-0-0-1-0$ & 1098.6504 & $1098.6530^{\mathrm{c}}$ & 68 \\
\hline
\end{tabular}

${ }^{\mathrm{a}}$ All accurate mass measurements were performed in positive mode ESI-MS, for either ${ }^{\mathrm{b}}$ $[\mathrm{M}+\mathrm{H}]^{+}$or $^{\mathrm{c}}\left[\mathrm{M}+\mathrm{NH}_{4}\right]^{+}$

All the oligomers composing these libraries were first individually analyzed by electrospray ionizationhigh resolution mass spectrometry (ESI- HRMS). In all cases, the main signal could be assigned to the desired oligomer based on elemental composition derived from accurate measurements performed in the positive ion mode (Table 1).Moreover, all samples 
were highly monodisperse, although some impurities were sometimes detected as shown by mass spectra recorded in the negative ion mode (Figures S1-S6). Furthermore, the monomer sequence of each digital oligourethane was confirmed by negative mode ESI-MS/MS (Figures S7-S12).

As mentioned in the introduction, three model commodity polymers were tested as host-matrices for the 2D-coded oligourethane libraries, namely polystyrene, poly(vinyl chloride) and polyethylene terephthalate. In order to show the versatility of the 2D-barcoding concept, both the three-component and the four-component oligourethane libraries were tested for labelling these three different polymers (i.e. six different samples were studied).For optimal anti-counterfeiting and traceability applications, the amount of barcodes that is included inside a product shall be as low as possible in order to not alter the physico-chemical properties of the host materials and to not be too easily spotted by potential infringers. Still, the embedded amount of the molecular barcode shall be sufficiently high to allow its extraction from the host matrix and its analytical detection. Following these prerequisites, the global oligourethane content was kept below $1 \mathrm{wt} \%$ in all studied films (i.e. $0.6-0.7 \mathrm{w} \%$ for the three-component library and $0.7-0.9 \mathrm{w} \%$ for the four component library). The sequencecoded libraries were incorporated in the polymer matrices using a simple solvent casting procedure. Typically, the host matrix and the oligourethanes constituting the chosen coded library were dissolved in a common good solvent(i.e. tetrahydrofuran for PS and PVC, phenol for PET) and mixed together. In most cases, it was necessary to heat the solutions in order to obtain a good dissolution of the oligourethanes, and eventually of the host matrix (e.g. for PET in phenol). The solutionswere then poured into glass plates and the solventswereevaporated to form homogeneous thin films.For PS and PVC, evaporation was performed at room temperature, whereas heating was necessary to form the PET films. It shall be noted that these mild thermal treatments (i.e. in the $50-90^{\circ} \mathrm{C}$ range) are not leading to noticeable oligourethane degradation. Indeed, as demonstrated in an earlier work, ${ }^{[25]}$ the 
thermal decomposition of sequence-coded oligourethanes starts to occur around $150^{\circ} \mathrm{C}$. In all cases, dried polymer films - transparent for PS and PVC and white for PET - were easily obtained via this solvent casting procedure.
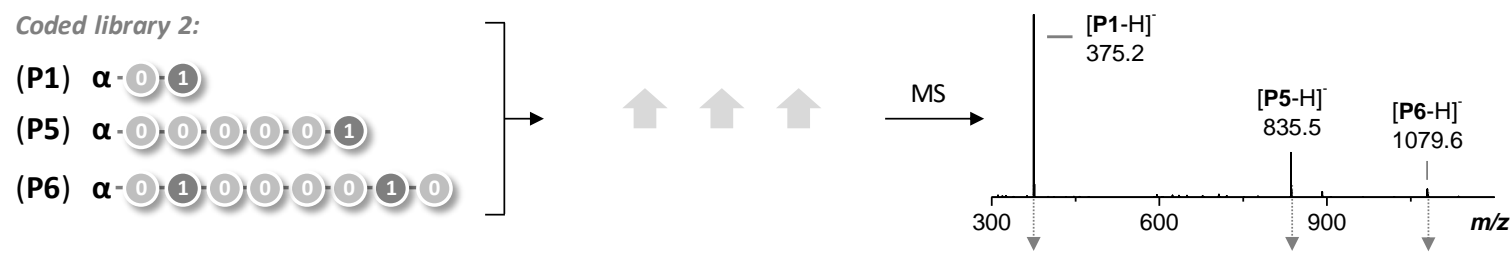

(a)

(b)

(c)

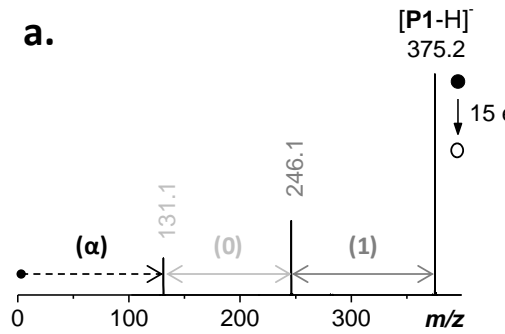

b.

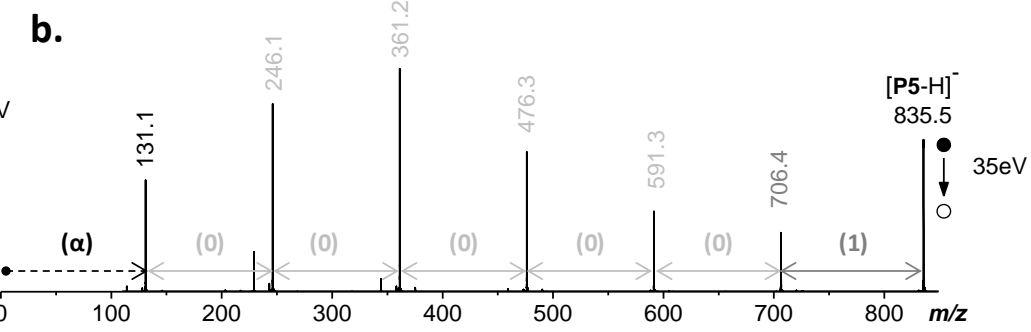

c.

[P6-H]

1079.6

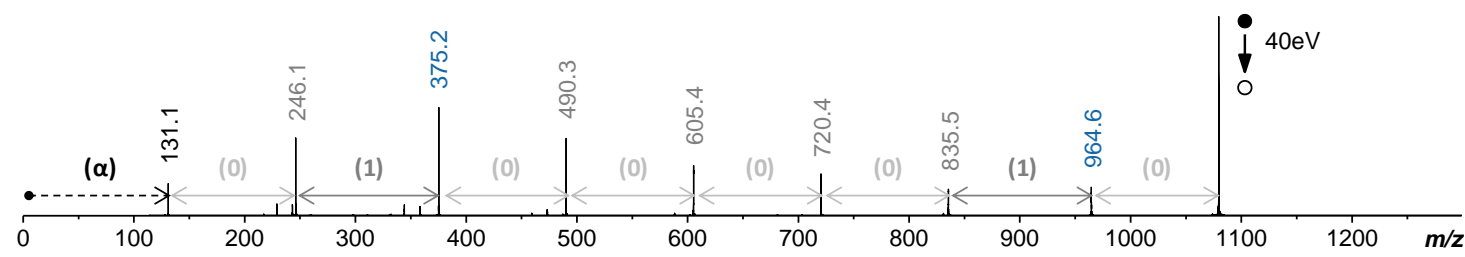

Figure 2.Mass spectrometry analysis of a PET membrane labeled with the threecomponent oligourethanes library. The three oligomers were first included in a PET film through a solvent casting procedure. Through selective methanol extraction, the oligomer mixture was extracted from the film and analyzed by ESI-MR (top left). All oligomers are detected in this spectrum and can therefore be individually sequenced by MS/MS. Panels $\mathbf{a}, \mathbf{b}$ and $\mathbf{c}$ shows the MS/MS spectra that were obtained,after solvent extraction from the PET films, for oligomers P1, P5 and P6, respectively

The formed films were then analyzed in order to verify the presence and the readability of the $2 \mathrm{D}$ barcodes within the host matrices. First, it was important to verify that the oligourethanes were homogeneously dispersed within the films. Taking into account the 
very low weight fraction and the very short chain-length of the oligourethanes that wereembeddedin the host matrices, phase segregation shall not occur within the films. Nevertheless, homogeneity was verified using a simple qualitative ${ }^{1} \mathrm{H}$ NMR analysis. For this, small pieces of the films were cut at different locations and placed in a deuterated solvent. It shall be noted that the chosen deuterated solvent shall not necessarily fully solubilize the host films but shall solubilize the oligourethanes. The PET and PVC membranes were studied in DMSO- $d_{6}$, whereas the PS membrane was studied in THF- $d_{6}$.For all types of host matrices, the NMR spectra indicated the presence of the oligourethanes in all studied regions of the films, thus ruling out the possibility of an inhomogeneous distribution(Figures S13-S15).More importantly, the possibility to extract the 2D-oligourethane mixtures from the films was studied. To do so, all films were incubated in a methanolic solution of ammonium acetate (3 $\mathrm{mM}$ ) for $10 \mathrm{~min}$. Methanol is a non-solvent for PS, PVC and PET but it partially solubilizes oligourethanes at room temperature. Composition of this incubation solution allows optimal ionization of oligourethanes in the negative ion mode and was hence specifically selected to enable direct injection of extracts in the ESI source with no prior dilution. Figure 2 shows the MS spectrum that was obtained for the PET membrane labeled with the three-component oligourethanes library. It appears clearly that the three oligomers P1, P5 and P6 of the library can be clearly identified as deprotonated species $[\mathrm{M}-\mathrm{H}]^{-}$at $\mathrm{m} / \mathrm{z}$ values of $375.2,835.5$ and 1079.6, respectively. Due to their intentional mass differences, these three oligomers lead to a detectable first-dimension fingerprint in $\mathrm{MS} \cdot{ }^{[28]}$ Furthermore, each peak of this mass distribution can be individually sequenced by MS/MS. Figure 2a-c shows the MS/MS spectra that were obtained for the three oligomers. Their digital sequence can be very easily deciphered, showing that neither solvent casting nor matrix-storage affected their structural integrity. In the present case, the model protection sequence 0100000101000010 was unequivocally found, as evidenced in Figure 2.Comparable results were obtained for all types of films and all coded libraries (Figures S16-S20). It shall be mentioned that MS spectra 
obtained for PVC films showed oligourethanes signals of with much lower intensity compared to the other two membranes and exhibited noticeable impurities, which were initially present in the commercial PVC samples as supported by MS data recorded for the extract of a non-labeled PVC film (Figure S21).

\section{Conclusions}

In summary, uniform sequence-coded oligourethaneswere used as anti-counterfeit tags in commodity polymer matrices, i.e. PS, PVS and PET. However, instead of using one type of coded oligomer as reported before, ${ }^{[27]}$ multicomponent libraries containing oligomers with different chain-length, sequence and mass were used. These coded libraries could be dispersed in all types of polymer matrices using facile solvent casting procedures. Furthermore, the oligomer mixtures could be extracted in all cases from the films and analyzed by mass spectrometry. In particular, a combination of MS and MS/MS analysis allows to reconstruct a complex digital sequence included in the whole library. These results are important because they indicate that oligourethane barcoding is not restricted to singlecoded-chains but can be extended to more complex oligomer mixtures. Furthermore, the results of this communication evidence that the concept is relatively universal and can be applied to very different types of host plastics. Indeed, the selective solvent-extraction protocols described in this work are practicaland allow direct MS analysis of the sequencecoded barcodes (i.e. without requiring barcode amplification or other pre-analysis treatments).These results open up interesting new horizons for the identification and traceability of plastic-based or plastic-containing products.

\section{Supporting Information}

Supporting Information is available from the Wiley Online Library or from the author marc201700426-sup-0001-S1.pdf 


\section{Acknowledgements}

J.F.L. thanks the H2020 program of the European Union (project Euro-Sequences, H2020MSCA-ITN-2014, grant agreement $n^{\circ} 642083$ ) and the CNRS for financial support. The PhD position of D. K. is supported by the ITN Euro-Sequences. L.C. acknowledges support from Spectropole, the Analytical Facility of Aix-Marseille University, by allowing a special access

to the instruments purchased with European Funding (FEDER OBJ2142-3341).The SEC results shown in the publication were obtained with the help of the polymer characterization service of the Institut Charles Sadron. 


\section{References}

[1] J.-F. Lutz, M. Ouchi, D. R. Liu, M. Sawamoto, Science 2013, 341, 1238149.

[2] J.-F. Lutz, Macromolecules 2015, 48, 4759-4767.

[3] Note that term "barcode" is only used here as a practical way to denote an identification marker. Indeed, coded sequences in polymers do not rely on width and spacing differences as macroscopic barcodes do.

[4] R. N. Grass, R. Heckel, M. Puddu, D. Paunescu, W. J. Stark, Angew. Chem., Int. Ed. 2015, 54, 2552-2555.

[5] D. Paunescu, W. J. Stark, R. N. Grass, Powder Technol. 2016, 291, 344-350.

[6] S. Pfeifer, Z. Zarafshani, N. Badi, J.-F. Lutz, J. Am. Chem. Soc. 2009, 131, 9195-9196.

[7] T. T. Trinh, L. Oswald, D. Chan-Seng, J.-F. Lutz, Macromol. Rapid Commun. 2014, $35,141-145$.

[8] A. Al Ouahabi, L. Charles, J.-F. Lutz, J. Am. Chem. Soc. 2015, 137, 5629-5635.

[9] A. Al Ouahabi, M. Kotera, L. Charles, J.-F. Lutz, ACS Macro Lett. 2015, 4, 10771080 .

[10] R. K. Roy, A. Meszynska, C. Laure, L. Charles, C. Verchin, J.-F. Lutz, Nat. Commun. 2015, 6, 7237.

[11] G. Cavallo, A. Al Ouahabi, L. Oswald, L. Charles, J.-F. Lutz, J. Am. Chem. Soc. 2016, $138,9417-9420$.

[12] M. Porel, C. A. Alabi, J. Am. Chem. Soc. 2014, 136, 13162-13165.

[13] C. Proulx, S. Yoo, M. D. Connolly, R. N. Zuckermann, J. Org. Chem. 2015, 80, 10490-10497.

[14] S. Martens, J. Van den Begin, A. Madder, F. E. Du Prez, P. Espeel, J. Am. Chem. Soc. 2016, 138, 14182-14185.

[15] S. C. Solleder, D. Zengel, K. S. Wetzel, M. A. R. Meier, Angew. Chem., Int. Ed. 2016, 55, 1204-1207.

[16] J.-F. Lutz, J.-M. Lehn, E. W. Meijer, K. Matyjaszewski, Nat. Rev. Mater. 2016, 1, 16024.

[17] H. M. Colquhoun, J.-F. Lutz, Nat. Chem. 2014, 6, 455-456.

[18] H. Mutlu, J.-F. Lutz, Angew. Chem., Int. Ed. 2014, 53, 13010-13019.

[19] L. Charles, C. Laure, J.-F. Lutz, R. K. Roy, Macromolecules 2015, 48, 4319-4328.

[20] J.-A. Amalian, T. T. Trinh, J.-F. Lutz, L. Charles, Anal. Chem. 2016, 88, 3715-3722. 
[21] L. Charles, G. Cavallo, V. Monnier, L. Oswald, R. Szweda, J.-F. Lutz, J. Am. Soc. Mass. Spectrom. 2017, 28, 1149-1159.

[22] C. Cho, E. Moran, Cherry, J. Stephans, S. Fodor, C. Adams, A. Sundaram, J. Jacobs, P. Schultz, Science 1993, 261, 1303-1305.

[23] P. A. Wender, J. B. Rothbard, T. C. Jessop, E. L. Kreider, B. L. Wylie, J. Am. Chem. Soc. 2002, 124, 13382-13383.

[24] R. L. Kanasty, A. J. Vegas, L. M. Ceo, M. Maier, K. Charisse, J. K. Nair, R. Langer, D. G. Anderson, Angew. Chem., Int. Ed. 2016, 55, 9529-9533.

[25] U. S. Gunay, B. E. Petit, D. Karamessini, A. Al Ouahabi, J.-A. Amalian, C. Chendo, M. Bouquey, D. Gigmes, L. Charles, J.-F. Lutz, Chem 2016, 1, 114-126.

[26] T. T. Trinh, C. Laure, J.-F. Lutz, Macromol. Chem. Phys. 2015, 216, 1498-1506.

[27] D. Karamessini, B. E. Petit, M. Bouquey, L. Charles, J.-F. Lutz, Adv. Funct. Mater. 2017, 27, 1604595.

[28] C. Laure, D. Karamessini, O. Milenkovic, L. Charles, J.-F. Lutz, Angew. Chem., Int. Ed. 2016, 55, 10722-10725. 